See discussions, stats, and author profiles for this publication at: https://www.researchgate.net/publication/220233767

\title{
Stochastic Analysis of Finite Population Bufferless Multiplexing in Optical Packet/Burst Switching Systems
}

Article in IEICE Transactions on Communications · February 2007

DOI: 10.1093/ietcom/e90-b.2.342 · Source: DBLP

CITATIONS

READS

15

52

2 authors:

Nail Akar

Bilkent University

103 PUBLICATIONS 765 CITATIONS

SEE PROFILE

Bahçeşehir University

22 PUBLICATIONS 166 CITATIONS

SEE PROFILE

Some of the authors of this publication are also working on these related projects:

Waste Management View project 


\title{
LETTER Stochastic Analysis of Finite Population Bufferless Multiplexing in Optical Packet/Burst Switching Systems
}

\author{
Nail AKAR ${ }^{\dagger \mathrm{a})}$, Member and Yavuz GUNALAY ${ }^{\dagger \dagger)}$, Nonmember
}

\begin{abstract}
SUMMARY In this letter, we study the blocking probabilities in an asynchronous optical packet/burst switching system with full wavelength conversion. Most of the existing work use Poisson traffic models that is well-suited for an infinite population of users. In this letter, the optical packet traffic arriving at the switching system is modeled through a superposition of a finite number of identical on-off sources. We propose a block tridiagonal LU factorization algorithm to efficiently solve the two dimensional Markov chain that arises in the modeling of the switching system.

key words: optical packet switching, optical burst switching, on-off traffic, Markov chains
\end{abstract}

\section{Introduction}

Two packet-based optical switching paradigms have recently been introduced for efficient transport of IP (Internet Protocol) traffic over WDM (Wavelength Division Multiplexing) networks: Optical Packet Switching (OPS) [1] and Optical Burst Switching (OBS) [2]. Although the control planes of OPS and OBS are different, they have similar data planes and to refer to a packet or burst we'll use the term "packet" in place of both.

In synchronous (time-slotted) optical packet switching, packet lengths are fixed and packets are assumed to arrive at slot boundaries. In asynchronous (unslotted) networks, optical packets are of variable size and packets arrive asynchronously eliminating the need for costly synchronization equipment. Our focus in this study is on the blocking probabilities in asynchronous optical packet switching systems. We also assume bufferless switching nodes in contrast with those that use Fiber Delay Lines (FDL) for buffering purposes.

When an optical packet arrives at an optical packet switch, it is switched towards the destination fiber over the incoming wavelength if that wavelength is available. Otherwise, a wavelength conversion unit is used to convert the incoming wavelength to one that is idle on the destination fiber. A packet is lost if there are no idle wavelengths (or channels) on the destination fiber. We assume full wavelength conversion in this let-

\footnotetext{
${ }^{\dagger}$ The author is with the Electrical and Electronics Engineering Department, Bilkent University, Ankara, Turkey

${ }^{\dagger \dagger}$ The author is with the Management Department, Bilkent University, Ankara, Turkey

a) E-mail: akar@ee.bilkent.edu.tr

b) E-mail: gunalay@bilkent.edu.tr
}

ter so a loss cannot be due to lack of a conversion unit [3]. When the packet arrival process is Poisson and the packet lengths are exponentially distributed then the model reduces to the well-known $\mathrm{M} / \mathrm{M} / \mathrm{c} / \mathrm{c}$ multiplexing model [4]. However in practice packets are generated by a relatively few number of sources, i.e., routers at the edge of an OBS network which invalidates the Poisson assumption. In this finite population case, each source is transmitting a variable length optical packet in the on state which is followed by an off state in which the source stays idle. The finite population Engset model [4] is not a proper fit for the problem under study since blocked packets would still continue to be dumped towards the network in optical packet switching networks due to one-way signaling. In [5] and [6], a two-dimensional Markov chain is studied to find the packet blocking probability when the on and off times are exponentially distributed. However, the authors do not take advantage of the special block structure of the arising Markov chain and it becomes very hard to study large systems, for example the largest system studied in [5] is fed with thirty three sources. In this letter, we provide a numerically efficient and algorithmic solution for the blocking probabilities for finite population bufferless multiplexing with exponentially distributed source on and off times. In addition, we study the impact of the distribution of on and off times on system performance by simulations.

\section{Mathematical Model}

We study an optical packet switch which has a number of input and output fibers and $W$ wavelength channels per fiber. Consider an output fiber, say $f$, of the switch. We assume $K$ sources sending optical packet traffic destined for fiber $f$. Let the on and off periods of each source be exponentially distributed with common means $1 / \mu$ and $1 / \lambda$, respectively. The mean offered load to the system is

$$
\rho=\frac{K \lambda}{W(\lambda+\mu)} .
$$

A new packet arriving at the input fibers of the switch and that is destined to output fiber $f$ is assumed to be blocked only due to output contention, i.e., all the $W$ channels on fiber $f$ are in use, and not due to the switching fabric itself. This assumption is valid if one 
uses a non-blocking optical switching fabric such as the bufferless broadcast and select architecture described in [7]. The source whose packet gets blocked would never be informed of the loss and would continue to dump its packet toward the switch.

Let $i(t)$ and $j(t)$ denote the number of wavelength channels that are in use and the number of sources that are in the on state, respectively. The process $\{(i(t), j(t)): t \geq 0\}$ is then a Markov process on the state space $S=\{(i, j): 0 \leq j \leq K, 0 \leq i \leq \min (W, j)\}$. To show this, let us assume that the process is in some state $(i, j)$ at time $t$. If a new packet arrives in the interval $(t, t+\delta t)$ which occurs with probability $(K-j) \lambda \delta t+o(\delta t)$ (i.e., $\left.\lim _{\delta t \rightarrow 0} o(\delta t) / \delta t=0\right)[8]$, then the packet will be admitted into the system if $i(t)<W$ and the Markov chain will jump to state $(i+1, j+1)$ or will be blocked when $i(t)=W$ and the visited state will be $(i, j+1)$. If a packet departure occurs in the interval $(t, t+\delta t)$ which occurs with probability $j \mu \delta t+o(\delta t)$, then the Markov chain will jump to state $(i-1, j-1)$ with probability $\frac{i}{j}$ or to $(i, j-1)$ with probability $1-\frac{i}{j}$. This shows that the underlying system is Markov. The state space is then decomposed into subsets called levels such that the number of sources in the on state is constant within a level. The states are then enumerated in the following order:

$$
\begin{aligned}
S= & \{\underbrace{(0,0)}_{\text {level } 0}, \underbrace{(0,1),(1,1)}_{\text {level } 1}, \\
& \underbrace{(0,2),(1,2),(2,2)}_{\text {level } 2}, \cdots, \underbrace{(0, K), \cdots,(W, K)}_{\text {level } K}\} .
\end{aligned}
$$

Based on this enumeration, state transitions occur among neighbouring levels and the process is then written as a Continuous-Time Markov Chain (CTMC) with a block-tridiagonal infinitesimal generator $Q$ of the following form:

$$
Q=\left[\begin{array}{ccccc}
A_{0} & U_{1} & & & \\
D_{0} & A_{1} & U_{2} & & \\
& D_{1} & A_{2} & \ddots & \\
& & \ddots & \ddots & U_{K} \\
& & & D_{K-1} & A_{K}
\end{array}\right]
$$

Steady-state probabilities of this CTMC can be found by solving for the unique stationary solution [8]

$$
x Q=0, \quad x e=1,
$$

where $e$ is a column vector of ones of suitable size. Note that the size of $Q$ is $(W+1)\left(K-\frac{W}{2}+1\right)$ and calculating the stationary solution using conventional methods like in [5] would be prohibitive especially for large systems, e.g., $K=256, W>>0$. Next we give a numerical solution procedure by taking advantage of the blocktridiagonal structure of the generator also given in [9] and [3]. Since one of the equations in (2) is redundant, we can replace one of the equations, say the first equation in (2). For this purpose, let $P$ be obtained by replacing the entries of the first column of $Q$ by setting $A_{0}=1, D_{0}=(1,1)^{T}$. Also let $b$ be a zero row vector except its first unity entry. It is clear that if $z$ is a solution to $z P=b$ then $x=\frac{z}{z e}$ gives the steady-state probabilities. We propose the block tridiagonal LU factorization algorithm given in [9] for solving $z P=b$. In this algorithm, the goal is to obtain a block LU factorization of the matrix $P$. For this purpose, we first partition the solution vectors $z=\left(z_{0}, z_{1}, \ldots, z_{K}\right)$, $x=\left(x_{0}, x_{1}, \ldots, x_{K}\right)$, and $b=\left(b_{0}, b_{1}, \ldots, b_{K}\right)$ according to levels. We then compute the matrices $\left\{F_{j}\right\}, j=$ $0,1, \ldots, K$ and $\left\{L_{j}\right\}, j=0,1, \ldots, K-1$ that comprise the block LU factorization using the following recurrence relation $[9]$ :

$$
\begin{aligned}
& F_{0}=A_{0} \\
& y_{0}=b_{0} F_{0}^{-1} \\
& \text { for } j=1 \ldots K \\
& \qquad L_{j-1}=D_{j-1} F_{j-1}^{-1} \\
& \qquad F_{j}=A_{j}-L_{j-1} U_{j} \\
& \qquad y_{j}=\left(b_{j}-y_{j-1} U_{j}\right) F_{j}^{-1} \\
& \text { end }
\end{aligned}
$$

By backward substitution, one can then find $z_{j}, j=0,1, \ldots, K$ :

$$
\begin{aligned}
& z_{K}=y_{K} \\
& \text { for } j=K-1 \ldots 0 \\
& \qquad z_{j}=y_{j}-z_{j+1} L_{j} \\
& \text { end }
\end{aligned}
$$

In the above algorithm, the computation intensive part is the $\mathrm{LU}$ decomposition of the matrices $\left\{F_{j}\right\}$ required in solving the linear systems in the block LU decomposition algorithm and the size of $F_{j}$ equals $j+1$ for $j \leq W$ and it is $W+1$ otherwise. An LU decomposition requires $2 / 3 N^{3}$ flops for an $N \times N$ matrix [9] and therefore the proposed algorithm requires $2 / 3(W+1)^{2}\left(W^{2} / 4+(W+1)(K-W+1)\right)$ flops for all the LU decompositions. Comparing with the brute force approach, this gain is significant.

We note that blocking occurs when an arriving packet finds the system in the state $(W, j)$. Defining the partition $x_{k}=\left(x_{0, k}, \ldots, x_{\min (k, W), k}\right)$, the blocking probability $P_{b}$ is then expressed as

$$
P_{b}=\frac{\sum_{j=W}^{K} x_{W, j}(K-j)}{K \frac{\mu}{\lambda+\mu}} .
$$

\section{Numerical Study}

We first consider an optical packet switching node with $W$ channels per fiber and set the number of users to $K=\eta W$ where $\eta \in(1.25,2,4)$. Without loss of generality, the mean optical packet length is set to unity 


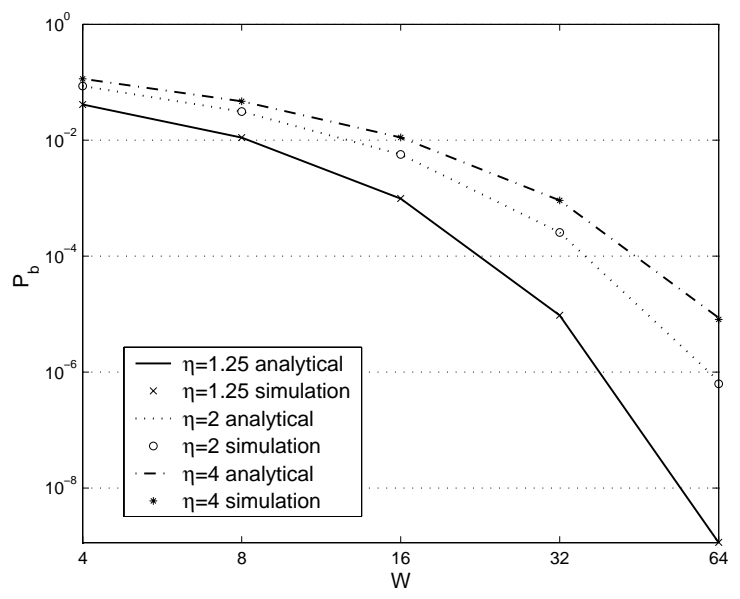

(a) $\rho=0.6$

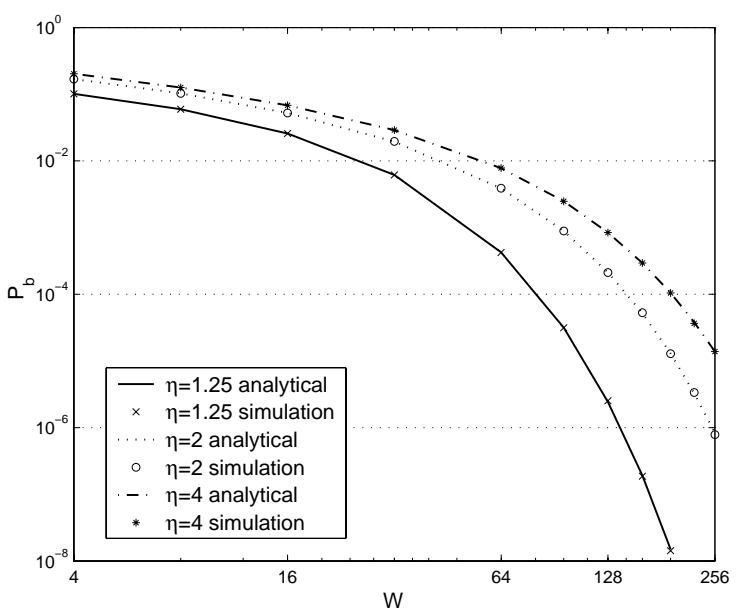

(b) $\rho=0.8$

Fig. 1 The packet blocking probability $P_{b}$ as a function of the number of wavelength channels $W$ for three different values of $\eta$ using analytical methods and simulations for the two cases (a) $\rho=0.6$ and (b) $\rho=0.8$.

throughout all the numerical examples. The blocking probabilities calculated by the proposed algorithm are compared against simulations in Fig. 1 for various $\rho$, $W$, and $\eta$, which demonstrates that the blocking probabilities obtained by the analytical method match the simulation results. Due to statistical multiplexing effect, we observe reduced blocking with respect to increasing number of channels. Moreover for a fixed $W$, we also observe that an increase in the number of users while the offered load is fixed, has an adverse effect on blocking performance.

For all the experiments above, the simulation results are obtained via the mean of twenty independent simulation runs. We also provide the $99 \%$ confidence intervals for our simulation experiment for $\rho=0.8$, $\eta=4$, and varying $W$ in Table 1 .

When $K \rightarrow \infty$ in a way that the total offered load is fixed, the input process is known to approach to the
Table 1 Comparison of analytical results with the $99 \%$ confidence intervals for the simulated system in terms of $P_{b}$ for the case $\rho=0.8$ and $\eta=4$.

\begin{tabular}{|c|c|c|}
\hline$W$ & Analytical & Simulation \\
\hline 4 & $2.022 \mathrm{E}-01$ & {$[2.022 \mathrm{E}-01,2.023 \mathrm{E}-01]$} \\
8 & $1.261 \mathrm{E}-01$ & {$[1.261 \mathrm{E}-01,1.261 \mathrm{E}-01]$} \\
16 & $6.807 \mathrm{E}-02$ & {$[6.805 \mathrm{E}-02,6.809 \mathrm{E}-02]$} \\
32 & $2.889 \mathrm{E}-02$ & {$[2.889 \mathrm{E}-02,2.895 \mathrm{E}-02]$} \\
64 & $7.808 \mathrm{E}-03$ & {$[7.804 \mathrm{E}-03,7.836 \mathrm{E}-03]$} \\
96 & $2.488 \mathrm{E}-03$ & {$[2.475 \mathrm{E}-03,2.489 \mathrm{E}-03]$} \\
128 & $8.434 \mathrm{E}-04$ & {$[8.392 \mathrm{E}-04,8.440 \mathrm{E}-04]$} \\
160 & $2.950 \mathrm{E}-04$ & {$[2.926 \mathrm{E}-04,2.951 \mathrm{E}-04]$} \\
192 & $1.051 \mathrm{E}-04$ & {$[1.036 \mathrm{E}-04,1.056 \mathrm{E}-04]$} \\
224 & $3.796 \mathrm{E}-05$ & {$[3.748 \mathrm{E}-05,3.816 \mathrm{E}-05]$} \\
256 & $1.384 \mathrm{E}-05$ & {$[1.356 \mathrm{E}-05,1.413 \mathrm{E}-05]$} \\
\hline
\end{tabular}

Poisson process with the same offered load. For the latter case, the blocking probability is described by the well-known Erlang loss formula [4]. To study this phenomenon, we fix $W=32$ and vary $K$ while fixing the offered load to the system. The blocking probability $P_{b}$ is calculated analytically and is depicted in Fig. 2 as a function of $K$ as well as the corresponding limiting results obtained using the Erlang loss formula. As expected, the blocking probability approaches as $K \rightarrow \infty$ to that found using the Erlang loss formula. However we also observe that the Erlang loss formula approximation for the finite population case is very conservative when the parameter $\eta=K / W$ is small, e.g., $\eta<4$.

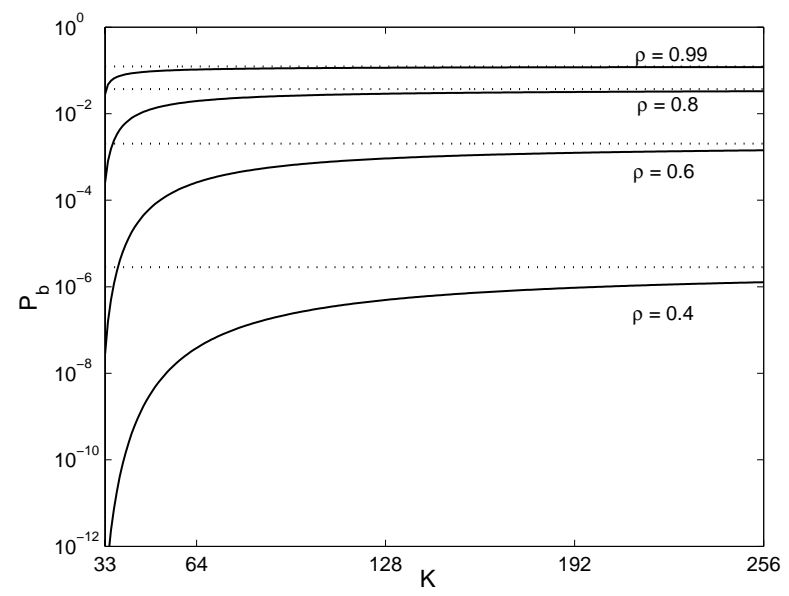

Fig. 2 The packet blocking probability $P_{b}$ using the proposed algorithm and the Poisson approximation when $W=32$ as a function of the number of users $K$ for four different values of the offered load. Solid and dotted curves are obtained using the proposed algorithm and the Erlang loss formula, respectively.

Table 2 presents our findings on the maximum achievable throughput $\rho\left(1-P_{b}\right)$ under two different grade of service requirements in terms of $P_{b}$ and for two different values of $W$ and varying values of $\eta$. Table 2 clearly shows that high throughputs in the order of 0.8 is achievable even without FDLs in the case of large systems, i.e., $W=32$, and for relaxed grade of 
Table 2 The maximum achievable throughput under two different grade of service requirements $P_{b}=10^{-2}$ and $P_{b}=10^{-4}$ as a function of $W$ and $\eta$.

\begin{tabular}{|c|c|c|c|c|}
\hline \multirow{2}{*}{$\eta$} & \multicolumn{2}{|c|}{$P_{b}=10^{-2}$} & \multicolumn{2}{c|}{$P_{b}=10^{-4}$} \\
\cline { 2 - 5 } & $W=8$ & $W=32$ & $W=8$ & $W=32$ \\
\hline 1.25 & .584 & .815 & .313 & .661 \\
\hline 1.50 & .527 & .780 & .271 & .613 \\
\hline 2 & .478 & .748 & .236 & .571 \\
\hline 4 & .425 & .711 & .202 & .524 \\
\hline 8 & .405 & .696 & .189 & .505 \\
\hline 16 & .396 & .689 & .183 & .497 \\
\hline
\end{tabular}

service requirements, i.e., $P_{b}=10^{-2}$. Such grade of service requirements are acceptable for best-effort data traffic that tolerates losses such as elastic TCP traffic. However, when the grade of service requirement gets stringent then the maximum achievable throughput drops substantially. It is also clear that the maximum achievable throughput is a decreasing function of $\eta$ and the actual number of optical packet users should also be taken into account for provisioning purposes. The more realistic case of non-exponential on and off times is hard to tackle analytically due to increased state space dimensionality. We now study the impact of on and off time distributions over blocking performance using simulations. For this purpose, we compare three models with the same average on and off times:

- EXP: Exponential on and off times.

- DEP-OFF: The optical packet lengths are still exponential but the off times are deterministically chosen based on the preceding packet length so that the offered load per user is fixed even for short time scales. In this sense, the off times are dependent on the on times. We believe that this model might be useful in optical packet switching networks with rate shaping at the edge.

- E4-ON: This model assumes exponential off times but $\mathrm{E}_{4}$-distributed on times where $\mathrm{E}_{4}$ denotes an Erlang 4 distribution with a squared coefficient of variation of $\frac{1}{4}$. This model can be used to model fixed packet length systems.

We simulate an optical packet switching system with $K=32$ and vary $W$ for three different offered loads. Table 3 compares the $99 \%$ confidence intervals for blocking probabilities for the models DEP-OFF and E4-ON with the EXP model for which we have exact solutions. We observe that the blocking probabilities for the EXP and DEP-OFF models are very close especially for high loads and the exact solutions for the former can safely be used to approximate the latter system. However, the EXP model comes short in accurately describing the E4-ON model and moreover we observe that increased determinism in the packet lengths reduces the blocking probabilities.
Table 3 The packet blocking probabilities using the model EXP and $99 \%$ confidence intervals for the models DEP-OFF and E4-ON when $K=32$ and for different values of $W$ and $\rho$.

\begin{tabular}{|c|c|c|c|c|}
\hline$W$ & $\rho$ & EXP & DEP-OFF & E4-ON \\
\hline 4 & .4 & $4.92 \mathrm{E}-02$ & {$[4.92 \mathrm{E}-02,4.93 \mathrm{E}-02]$} & {$[4.73 \mathrm{E}-02,4.74 \mathrm{E}-02]$} \\
12 & .4 & $8.13 \mathrm{E}-04$ & {$[8.12 \mathrm{E}-04,8.19 \mathrm{E}-04]$} & {$[7.20 \mathrm{E}-04,7.26 \mathrm{E}-04]$} \\
20 & .4 & $3.17 \mathrm{E}-06$ & {$[2.97 \mathrm{E}-06,3.25 \mathrm{E}-06]$} & {$[2.47 \mathrm{E}-06,2.69 \mathrm{E}-06]$} \\
4 & .6 & $1.27 \mathrm{E}-01$ & {$[1.27 \mathrm{E}-01,1.27 \mathrm{E}-01]$} & {$[1.20 \mathrm{E}-01,1.20 \mathrm{E}-01]$} \\
12 & .6 & $1.76 \mathrm{E}-02$ & {$[1.78 \mathrm{E}-02,1.79 \mathrm{E}-02]$} & {$[1.47 \mathrm{E}-02,1.47 \mathrm{E}-02]$} \\
20 & .6 & $1.35 \mathrm{E}-03$ & {$[1.38 \mathrm{E}-03,1.39 \mathrm{E}-03]$} & {$[9.77 \mathrm{E}-04,9.81 \mathrm{E}-04]$} \\
28 & .6 & $6.67 \mathrm{E}-06$ & {$[6.55 \mathrm{E}-06,6.80 \mathrm{E}-06]$} & {$[3.99 \mathrm{E}-06,4.08 \mathrm{E}-06]$} \\
4 & .8 & $2.16 \mathrm{E}-01$ & {$[2.15 \mathrm{E}-01,2.15 \mathrm{E}-01]$} & {$[2.00 \mathrm{E}-01,2.00 \mathrm{E}-01]$} \\
12 & .8 & $8.12 \mathrm{E}-02$ & {$[8.14 \mathrm{E}-02,8.16 \mathrm{E}-02]$} & {$[6.36 \mathrm{E}-02,6.37 \mathrm{E}-02]$} \\
20 & .8 & $3.17 \mathrm{E}-02$ & {$[3.21 \mathrm{E}-02,3.25 \mathrm{E}-02]$} & {$[2.02 \mathrm{E}-02,2.03 \mathrm{E}-02]$} \\
28 & .8 & $4.49 \mathrm{E}-03$ & {$[4.63 \mathrm{E}-03,4.64 \mathrm{E}-03]$} & {$[2.17 \mathrm{E}-03,2.18 \mathrm{E}-03]$} \\
\hline
\end{tabular}

\section{Conclusions}

In this paper, we study a finite population bufferless multiplexing problem which has immediate applications in the analysis and provisioning of bufferless optical burst/packet switching networks. The traffic is assumed to be generated from a superposition of a finite number of identical on-off sources. We reduce the problem of finding the blocking probabilities to the solution of a two dimensional CTMC in block tridiagonal form. Taking advantage of this structure, we employ a block LU factorization algorithm to efficiently find the blocking probabilities even for very large systems. Our findings show that the Erlang loss formula that can be used as an approximation to the finite population case is very conservative especially for small number of users. We also show that the blocking probabilities are sensitive to the on and off time distributions.

\section{References}

[1] G.N. Rouskas and L. Xu, "Optical packet switching," in Emerging Optical Network Technologies: Architectures, Protocols, and Performance, ed. K. Sivalingam and S. Subramaniam, pp.111-127, Springer, Norwell, Massachusetts, 2004.

[2] C. Qiao and M. Yoo, "Optical burst switching (OBS) - a new paradigm for an optical Internet," Jour. High Speed Networks (JHSN), vol.8, no.1, pp.69-84, 1999.

[3] N. Akar and E. Karasan, "Exact calculation of blocking probabilities for bufferless optical burst switched links with partial wavelength conversion," 1st Conference on Broadband Networks (BROADNETS'04), pp.110-117, 2004.

[4] V.B. Iversen, Teletraffic Engineering Handbook, ITU-D Study Group 2 Question 16/2, 2005.

[5] M. Zukerman, E.W.M. Song, Z. Rosberg, G.M. Lee, and H.L. $\mathrm{Vu}$, "On teletraffic applications to OBS," IEEE Communications Letters, vol.8, no.2, pp.116-118, 2004.

[6] H. Overby, "Performance modelling of optical packet switched networks with the Engset traffic model," OSA Optics Express, vol.13, no.5, pp.1685-1695, 2005.

[7] H. Buchta, C.M. Gauger, E. Patzak, and J. Saniter, "Limits of effective throughput of optical burst switches based on seminconductor optical amplifiers," Proceedings of the Optical Fiber Communication Conference (OFC), 2003.

[8] L. Kleinrock, Queuing Systems, Vol. 1, Theory, John Wiley, New York, 1989.

[9] G.H. Golub and C.F. van Loan, Matrix Computations, 3 ed., The Johns Hopkins University Press, 1996. 